\title{
Cognitive effects of androgen deprivation therapy in an older cohort of men with prostate cancer
}

\author{
Supriya Gupta Mohile ${ }^{a,{ }^{*}}$, Maureen Lacy ${ }^{b}$, Miriam Rodin $^{c}, K^{2}$ athryn Bylow ${ }^{d}$, William Dale ${ }^{e}$, \\ Michael R. Meager ${ }^{b}$, and Walter M. Stadler ${ }^{f}$ \\ a James Wilmot Cancer Center, University of Rochester, Rochester, NY, United States \\ b Department of Psychiatry, University of Chicago, Chicago, IL, United States \\ c Department of Medicine, Section of Geriatrics, St. Louis University, United States \\ d Department of Medicine, Division of Hematology/Oncology, Medical College of Wisconsin, \\ United States \\ e Department of Medicine, Division of Geriatrics and Palliative Care, University of Chicago, \\ Chicago, IL, United States \\ ${ }^{f}$ Department of Medicine, Division of Hematology/Oncology, University of Chicago, Chicago, IL, \\ United States
}

\begin{abstract}
Objective-To determine the baseline prevalence of cognitive impairment in older men treated with ADT and to assess changes in cognitive performance over time.

Methods and results-Thirty-two patients (median age of 71 years, range 51-87) were administrated an extensive neuropsychological testing battery prior to ADT initiation, with 21 (65\%) completing post-treatment evaluations 6 months later. At baseline, $45 \%$ scored $>1.5$ standard deviations below the mean on $\geq 2$ neuropsychological measures. Using standardized inferential statistics, no change in cognition was documented following treatment. The Reliable Change Index revealed that, on a case-by-case basis, $38 \%$ demonstrated a decline in measures of executive functioning and $48 \%$ showed improvement on measures of visuospatial abilities. Within exploratory analyses, patients who scored below expectation at baseline displayed no change in cognition, while patients with average or better scores at baseline displayed improvements in visuospatial planning and timed tests of phonemic fluency.
\end{abstract}

Conclusions-We found a high prevalence of lower than expected cognitive performance among a sample of patients just starting ADT for prostate cancer. Assessment of baseline cognitive function should be taken into account for future research and to inform clinical management.

\footnotetext{
* Corresponding author at: James P. Wilmot Cancer Center, 601 Elmwood Avenue, Box 704, Rochester, NY 14642, United States. Tel.: +1 585275 9319; fax: +1 585273 1042. supriya_mohile@urmc.rochester.edu (S.G. Mohile).

Reviewers

Shabbir M.H. Alibhai, MD, MSc, Staff Physician, University Health Network, Medicine, Room EN 14-214, 200 Elizabeth Street, Toronto, Ontario M5G 2C4, Canada.

Heather J. Green, Ph.D., Lecturer in Clinical Psychology, Griffith University, School of Psychology, Griffith University Gold Coast Campus, Gold Coast, QLD 4222, Australia.

Conflict of interest statement: The authors report no conflict of interests in the writing of this manuscript.
} 


\section{Keywords}

Cognition; Androgen deprivation; Prostate; Elderly

\section{Introduction}

Prostate cancer is currently the leading cancer diagnosis of men in the United States and is the second leading cause of male cancer-related death. The incidence of prostate cancer rapidly increases with age. Data from autopsy studies reveal that prostate cancer incidence rises from $>30 \%$ in men over 50 years to $80 \%$ by age 80 [1]. With increasing life expectancy, prostate cancer will become even more prevalent over the next few decades [1].

Androgen deprivation therapy (ADT) by medical or surgical castration (i.e., LHRH agonists or orchiectomy) is commonly combined with surgery or radiation options for locally advanced or high-risk prostate cancer. In addition, androgen ablation, with or without the concurrent use of peripheral anti-androgen therapies, is the mainstay of treatment for men with systemic disease, evident either by a rising prostate specific antigen (PSA) or overt metastatic disease. ADT has an initial response rate on the order of $85 \%$ and many men sustain long-term responses on therapy. For example, in a recent cooperative group trial, $43 \%$ of patients with metastatic bone disease achieved a PSA $\leq .2 \mathrm{ng} / \mathrm{ml}$ with ADT and their median survival was 75 months. These numbers are expected to be even higher in the larger group of patients in whom ADT is employed for biochemical recurrence only on the basis of controversial survival benefits [2-4].

As a result increasing attention is being paid to ADT toxicities, which include not only the highly discussed sexual dysfunction (e.g., decline in libido, difficulty initiating and maintaining erections), but also decreased bone mineral density, lean body mass and muscle strength and overall lower quality of life scores [5,6]. More recently, the effects of ADT on cognition have been evaluated. The reported studies have used a variety of methodologies and the results have been inconsistent. Nevertheless, a review by Nelson et al. of the published literature showed that between $47 \%$ and $69 \%$ of men on ADT declined in at least 1 cognitive domain [7]. The deficits were primarily noted within visuospatial abilities and executive functioning. However, none of the reviewed studies evaluated baseline cognitive performance before attributing poor performance on cognitive tests to ADT.

Like prostate cancer, the prevalence of cognitive impairment also increases with age. It is estimated that $6-10 \%$ of people 65 years or older suffer from some form of clinically significant dementia. The prevalence increases to $25-48 \%$ in samples of community-living populations over 80 years of age [8]. The prevalence of early or mild cognitive impairment (MCI) is estimated to be even higher. In one large longitudinal study, the prevalence of dementia was $13.9 \%$, while the prevalence of cognitive impairment without a defined dementia diagnosis was $22.2 \%$ in persons aged 71 years and over $[8,9]$. It is estimated that mild cognitive impairment is associated with an increased risk for progression to dementia, with progression rates of $10-15 \%$ per year as compared with $1-2.5 \%$ in persons who are cognitively intact at initial testing [9-13]. Of importance to note, the definition of mild cognitive impairment remains controversial but generally includes evaluation of performance on standard neuropsychological tests, but subjective memory loss symptoms, functional status, and imaging studies [13]. The high prevalence of MCI or dementia in community-dwelling older adult population suggests that elderly patients who are initiated on ADT may have a high prevalence of baseline cognitive dysfunction and may be at risk for further deterioration with such treatment. 
In the current study, we examined the neuropsychological functioning of a cohort of men diagnosed with prostrate cancer before and 6 months following ADT initiation to assess potential changes in cognitive abilities. Furthermore, we examined separately the impact of ADT on those who performed below expectation at baseline to determine if they are uniquely susceptible to ADT effects and thus may explain, in part, disparate findings in the literature.

\section{Methods}

This investigation reports an analysis of a longitudinal pilot study evaluating the potential neurocognitive effects of ADT in an older prostate cancer population. The study, conducted at the University of Chicago Hospitals, was approved by the Institutional Review Board and all patients provided written informed consent.

\subsection{Research participants}

The study population includes men over age 50 with histologically confirmed prostate cancer who were to undergo at least 6 months of androgen deprivation therapy with or without the addition of an anti-androgen as determined by their primary physician. Eligible patients could have any stage of disease, but those with overt metastatic disease were required to be asymptomatic. Other eligibility criteria included: (1) no clinically evident dementia or psychiatric impairment; (2) no current alcohol or drug abuse; (3) no previous cytotoxic or hormonal cancer treatment or planned treatment with chemotherapy for this malignancy; (4) English language proficiency; (5) no visual, hearing or physical impairment sufficient to interfere with neurocognitive testing; and (6) at least a 5th grade education level.

\subsection{Measures}

The protocol included a standardized neuropsychological battery detailed below. Baseline measures were administered within 2 weeks of starting androgen ablation and again approximately 6 months after starting treatment. Demographics and household characteristics were elicited from the patients. Cancer characteristics including stage and Gleason's score were abstracted from the charts.

Standard neuropsychological measures of attention, language, visuospatial planning, verbal memory, visual memory and motor processing were administered by a master's level trained psychometrician, under the supervision of an experienced neuropsychologist. Tests were selected for their established reliability, validity, and sensitivity. The test-retest variability for each of these tests is less than .5 standard deviations, typically established over an interval of several weeks. Alternate equivalent forms of several tests were used to adjust for learning effects. Administration typically took $1-2 \mathrm{~h}$ of time.

Attentional skills were assessed by the Trail Making Test and the Digit Span (forward and backward) subtest of the Wechsler Adult Intelligence Scales-III (WAIS-III). The Trail Making Test-A is a paper and pencil test requiring speeded sequencing of numbers on a page. The Trail-Making Test-B adds the additional demand of alternating between numbers and letters and thus requiring cognitive flexibility [14]. The Digit Span test requires individuals to repeat back numbers of increasing length, first forward and then backward. The Controlled Oral Word Association Test (COWAT) a gross language task, requires an individual to generate words to phonemic cues within a 1 min time frame with three trials (e.g., F, A and S or C, F, and L) [15]. Semantic fluency was also measured by the COWAT and measured the number of animals able to be named in 1 min [15]. The Rey Complex Figure Test-Copy Trial (RFT) assesses executive visuospatial planning abilities. The subject 
is asked to copy a complex figure from a drawing. The score assesses planning and accuracy [16]. The Hopkins Verbal Learning Test-Revised (HVLT-R) is utilized to measure verbal memory and the Brief Visual Spatial Learning Test-Revised (BVMT-R) measures visual memory. The HVLT-R measures immediate and delayed recall, learning with repetition, and recognition memory for a 12 -item word list $[17,18]$. The BVMT-R measures immediate and delayed recall, learning with repetition and visual recognition memory. It is psychometrically equivalent to the HVLT-R, so that verbal and visual memory can be compared [19]. The Grooved Pegboard and Finger Tapping Tests assess motor speed and dexterity. These are sensitive tests of fine motor coordination $[15,20]$.

As anxiety and depression have been documented to influence cognition in the elderly, participants also completed two questionnaires assessing mood state. The Beck Depression Inventory-2 (BDI-2) is brief, validated in the elderly and yields a severity of symptoms score $[21,22]$. It is less dependent on physical symptoms, which are common in the elderly, yet may not reflect depressive states (i.e., insomnia). The State Trait Anxiety Inventory (STAI) assesses for acute symptoms of anxiety, which may hinder performance, along with more characteristic traits of anxiety [23].

\subsection{Statistical analysis}

Our goal sample size was 30 patients who would complete both assessments. This sample size would provide $90 \%$ confidence to detect a decline of $>1.5$ standard deviations on 2 or more neuropsychological measures in $20 \%$ of patients within $\pm 12 \%$.

Each domain specific scale was summarized for mean values and standard deviations for each point-in-time measurement. Neuropsychological scores were compared for withinsubject changes from baseline to 6-months using paired $t$-tests. An impaired performance on a test was defined as scoring more than 1.5 standard deviations below the age and educationadjusted population reference mean for that test.

In addition to the aforementioned analyses, a Reliable Change Index (RCI) was computed for each individual's pre- and post-test scores for each neuropsychological measure employed in the study. The RCI is used to determine whether the magnitude of change for a subject is statistically reliable [24,25]. In other words, reliable change addresses whether the change is of sufficient magnitude to be confident that the change is beyond that which could be attributed to measurement error. By employing Jacobson and Truax's method [25], the pre-existing test-retest reliability coefficient (rxx) of each neuropsychological measure was used to calculate each measure's Standard Error (SE), which was calculated as $\mathrm{SD}_{1}(\sqrt{1}-$ rxx). SD1 was in turn used to compute the Standard Difference (SD) for each

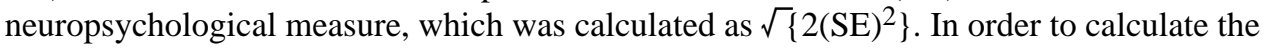
$\mathrm{RCI}$, the post-test score was subtracted from the pre-test score and subsequently divided by the Standard Difference (SD) [25]. An RCI value was considered significant if it was greater than or equal to \pm 1.64 , which established a $90 \%$ confidence interval around each significant RCI value [26]. Values that were positively greater than or equal to +1.64 demonstrated reliable improvement, while values that were less than or equal to -1.64 demonstrated reliable decline in test performance.

For all analyses, statistical significance was set at the conventional $p<.05$. The demographic and neuropsychological analyses were performed using SPSS version 15.0. STATA version 8.0 was used to examine general health and functional assessment measures. 


\section{Results}

A total of 32 subjects were recruited with a median age of 71.0 and a median of 15 years of education (Table 1). Twenty-one patients completed the second 6-month evaluation. The most common reason for not completing the second assessment was unwillingness to participate due to long time commitment (60\%), loss to follow-up (20\%), and funding or administrative issues (15\%). One patient died due to a comorbid illness prior to the second assessment.

Seventy-five percent of patients were over the age of 60 years with $50 \%$ over the age of 70 . Over one-third of the group was African American (34\%). Table 2 presents raw test data $(n$ $=32$ ) along with the percent of patients whose baseline scores were more than 1.5 standard deviations below the standardized population mean for the test. As a group, patients' performance on the delayed verbal memory task fell 1.5 standard deviations below normative data, suggestive of mild cognitive inefficiencies in verbal recall prior to treatment (i.e., "word finding difficulty"). Exploratory analyses revealed that $52 \%$ of the patients ( $n=$ 16) performed $>1.5$ standard deviation below the mean on a verbal or visual delayed memory measure. Included within the diagnostic criteria for MCI is performance below expectation on at least 1 standardized measure of cognitive function [13,27-29]. More importantly $45 \%$ met this criterion on two or more tests, typically memory plus executive tasks, which meets neuropsychological criteria for possible cognitive impairment. A full functional and clinical assessment would be needed to make a clinical diagnosis of MCI or dementia.

Table 3 presents results from paired $t$-tests used to assess change in cognitive test results over 6 months. No significant change was observed for the group as a whole on any of the cognitive measures, although motor speed was slightly faster at Time 2. A statistically significant increase in symptoms was documented on the depression inventory, albeit still below defined cutoffs for clinical depression for this population.

Given the recent concerns of relying on standard inferential statistics to evaluate treatment effects [30], we also utilized the RCI to improve assessment of within treatment variability of out-come. Using the RCI, none of the subjects significantly declined, $14 \%$ significantly improved, and 57\% showed a mixture of effects (both decline and improvement) on one or more cognitive measures. Examination of RCI on specific areas of cognition revealed the most consistent finding was improved visuospatial planning following treatment (Table 4).

Table 5 documents an exploratory analysis comparing the 6-month follow-up scores of patients who performed $>1.5$ standard deviations below expected on one or more measures at baseline $(n=11)$ with those who performed within expected ranges on all tests $(n=10)$. Again, impairment was defined as $>1.5$ standard deviations below the mean on the delayed memory recall trial. Analysis revealed no significant change in cognition for the low scoring group over 6 months, while the intact group displayed improved visuospatial planning ( $p=$. $01)$ and phonemic fluency $(p=.03)$.

\section{Discussion}

Prostate cancer is highly prevalent in older men. ADT is commonly utilized for these men in combination with other therapies for localized disease and long-term for systemic disease. In the present study, we examined the cognitive functioning of patients with recently diagnosed prostate cancer prior to ADT and again 6 months later. At baseline, prior to initiation of treatment, over half of our sample displayed memory impairment (e.g., >1.5 standard deviations below the normative mean on the delayed verbal recall test) $[9,27]$. Other studies have suggested that the prevalence of some level of cognitive impairment prior to treatment 
prior to treatment in other cancers may be as high as $20-30 \%$ [31]. Further exploration determined that $45 \%$ met the psychometric criteria for dementia, with a profile of memory impairment and often executive deficits [8]. While age and education are clearly contributors to cognitive performance, these variables may not entirely account for the prevalence of low scores in this group. We examined depression and anxiety as potential confounders, but they were unlikely to have accounted for our findings. Although there is evidence of cognitive impairment on neuropsychological testing, clinical evaluation including evidence of subjective memory complaints and evidence of functional impairment would be necessary to meet the clinical criteria for MCI or dementia [13]."

Consistent with Jenkin's et al. findings [26], no decline in any cognitive domain was documented following treatment. However, RCI documented improved visuospatial planning post-treatment. Furthermore, an exploratory analysis revealed that after 6 months of ADT, those who scored $>1.5$ standard deviations below expected on any of the scales at baseline had no changes in performance. However, persons with normal scores on every test at baseline demonstrated improvements on visuospatial planning measures of executive control and phonemic fluency tasks. It is possible that improvement represents learning and practice effects on the part of these men compared with the absence of learning among men who performed worse at baseline.

A review of the literature on the cognitive effects of hormone therapy in men with prostate cancer have conflicting results due to small sample size, short observation time, or perhaps a heterogeneous effect of ADT [7]. While practice effects may have played a role masking mild decline for some patients, the low performing group at baseline failed to demonstrate this benefit. The lack of practice effects in the completion of neuropsychological assessments may be itself suggestive of impairment [15,32]. While the sample size is small, this study suggests that future research should measure cognitive status at baseline before assessing cognitive response to treatment.

Several studies have evaluated effects of ADT on the cognition of men with prostate cancer but few have described baseline prevalence of cognitive impairment in this population. Salminen et al. examined the cognitive functioning of 25 men, ages 49-75, following 12 months of androgen deprivation [33]. They found improvements in visual and semantic memory. While not significantly different, the range on the Mini-Mental State Exam (MMSE) of the treatment group at baseline was 21-29, suggesting that at least some of the patients had significant cognitive impairment prior to initiation of treatment. In fact, further analysis between groups revealed significant differences on several attentional tasks prior to initiation of treatment. In a study of outcomes at 1 year, Green et al. again found cognitive losses in those undergoing hormonal treatment compared to controls [34]. They also noted that the community control group performed significantly better than the prostate cancer group at baseline $(p=.002)$. This again may suggest the possibility that some subset of patients may have cognitive impairment prior to treatment which may impact overall results. Jenkins et al. examined 36 patients and 25 controls on several cognitive tasks prior to and following treatment [26]. While they found no group differences using ANOVA analysis, RCI analysis revealed $47 \%$ of patients declined on at least one task at 6 months. The most commonly affected tasks involved visuospatial processing. Despite using age and IQ matched controls, the baseline cognitive performance of the patient group is unclear. As the authors pointed out, group comparisons can sometimes miss significant differences. Thus, the role that prior cognitive status may have on mediating any effect of hormonal treatment on cognition is still unclear.

There were limitations in this study that could have impacted results. Many of our men did not complete the follow-up assessment. This drop-out was mostly due to the time 
commitment for the procedure and difficulty with scheduling in concert with other appointments. Those who did not complete the follow-up assessment may be different than those that did, although baseline patient and disease characteristics were not significantly different between the two groups (data not shown). Our sample size was small, and exploratory analyses may have resulted in spurious associations as a result. A control group was not utilized in this study, instead each individual acted as his own control. Although we utilized different versions of several of the neuropsychological tests at each time point, practice effects were not explicitly controlled for. Lastly, our follow-up time of 6 months between testing is short and future work should evaluate the cognitive function of prostate cancer patients on ADT over a longer time period. Despite these limitations, this study is the first to directly evaluate the potential differential impact of ADT on the cognition of older men relative to their baseline differences in cognitive functioning.

In summary, the current study documented that at baseline close to half of the subjects scored more than 1.5 standard deviations below expected on one or more key cognitive tasks, verbal memory and executive function. Initial deficits may be due to co-existing medical conditions such as vascular disease. If cerebrovascular small vessel disease progresses, it may present as subcortical dementia unrelated to any underlying malignancy or therapy. Longer studies hopefully will provide further etiological and prognostic clarification. In this study, none of the subjects had a clinical diagnosis of cognitive impairment recognized by the treating physicians. Although baseline cognitive impairment may be subclinical and difficult to detect, other patients may score as impaired on screening tests for cognition, which could be utilized efficiently in the clinical oncology setting. For example, our research team noted previously that $24 \%$ of older prostate cancer patients on androgen deprivation therapy scored as "impaired" on a short cognitive screening test, the Short Portable Mental Status Questionnaire [35]. Based on these findings, it may be important for clinicians to assess initial cognitive status when discussing treatment options with patients and their ability to execute treatment plans. Furthermore, these patients may be especially vulnerable to developing a more pervasive and life altering condition such as dementia and thus should receive psychoeducational services and assessment from a multidisciplinary team of cognitive specialists to explore treatment options. These findings also suggest that assessment of cognitive performance at baseline may be important in the research setting if there is collection of self-report data or if the proposed treatment options and plans are complex. Future studies should document the pre-treatment cognitive status of older prostate cancer patients and subsequent vulnerability after hormonal interventions.

\section{Acknowledgments}

The authors did not have any writing assistance. Funding sources include the American Society of Clinical Oncology Young Investigator Award and the Hartford Geriatrics Health Outcomes Scholar Award to Supriya Mohile. Study sponsors had no role in the writing of the manuscript and in the decision to submit the manuscript for publication.

\section{Biography}

Supriya Gupta Mohile, M.D., M.S. is a geriatric oncologist whose research focuses on the quality of life and health outcomes of vulnerable and frail older adults with cancer. She is an Assistant Professor at the University of Rochester's James Wilmost Cancer Center and was the recipient of an American Society of Clinical Oncology Young Investigator Award and a Hartford Geriatrics Health Outcomes Research Award. Her clinical expertise focuses on the care of older patients with genitourinary and gastrointestinal malignancies. 


\section{References}

1. Beedassy A, Cardi G. Chemotherapy in advanced prostate cancer. Semin Oncol. 1996; 26:428-38. [PubMed: 10482185]

2. Bolla M, Gonzalez D, Warde $\mathrm{P}$, et al. Improved survival in patients with locally advanced prostate cancer treated with radiotherapy and goserelin. N Engl J Med. 1997; 337(5):295-300. [PubMed: 9233866]

3. Messing EM, Manola J, Yao J, et al. Immediate versus deferred androgen deprivation treatment in patients with node-positive prostate cancer after radical prostatectomy and pelvic lymphadenectomy. Lancet Oncol. 2006; 7(6):472-9. [PubMed: 16750497]

4. Granfors T, Modig H, Damber JE, Tomic R. Combined orchiectomy and external radiotherapy versus radiotherapy alone for nonmetastatic prostate cancer with or without pelvic lymph node involvement: a prospective randomized study. J Urol. 1998; 159(6):2030-4. [PubMed: 9598512]

5. Basaria S, Dobs AS. Risks versus benefits of testosterone therapy in elderly men. Drugs Aging. 1999; 15(2):131-42. [PubMed: 10495072]

6. Basaria S, Lieb J 2nd, Tang AM, et al. Long-term effects of androgen deprivation therapy in prostate cancer patients. Clin Endocrinol (Oxf). 2002; 56(6):779-86. [PubMed: 12072048]

7. Nelson CJ, Lee JS, Gamboa MC, Roth AJ. Cognitive effects of hormone therapy in men with prostate cancer: a review. Cancer. 2008; 113(5):1097-106. [PubMed: 18666210]

8. Plassman BL, Langa KM, Fisher GG, et al. Prevalence of dementia in the United States: the aging, demographics, and memory study. Neuroepidemiology. 2007; 29(1-2):125-32. [PubMed: 17975326]

9. Plassman BL, Langa KM, Fisher GG, et al. Prevalence of cognitive impairment without dementia in the United States. Ann Intern Med. 2008; 148(6):427-34. [PubMed: 18347351]

10. Kelley BJ, Petersen RC. Alzheimer's disease and mild cognitive impairment. Neurol Clin. 2007; 25(3):577-609. v. [PubMed: 17659182]

11. Petersen RC. Mild cognitive impairment as a diagnostic entity. J Intern Med. 2004; 256(3):183-94. [PubMed: 15324362]

12. Petersen RC. Mild cognitive impairment: current research and clinical implications. Semin Neurol. 2007; 27(1):22-31. [PubMed: 17226738]

13. Petersen RC, Doody R, Kurz A, et al. Current concepts in mild cognitive impairment. Arch Neurol. 2001; 58(12):1985-92. [PubMed: 11735772]

14. Reitan RM. The relation of the trail making test to organic brain damage. J Consult Psychol. 1955; 19(5):393-4. [PubMed: 13263471]

15. Lemay S, Bedard MA, Rouleau I, Tremblay PL. Practice effect and test-retest reliability of attentional and executive tests in middle-aged to elderly subjects. Clin Neuropsychol. 2004; 18(2): 284-302. [PubMed: 15587675]

16. Meyers, J.; Meyers, K. Professional Manual. Palo Alto: Psychological Assessment Resources; 1995. Rey Complex Figure Test and Recognition Trial.

17. Brandt J. The Hopkins Verbal Learning Test: development of a new memory test with six equivalent forms. Clin Neuropsychol. 1991; 5:125-42.

18. Shapiro AM, Benedict RH, Schretlen D, Brandt J. Construct and concurrent validity of the Hopkins Verbal Learning Test-revised. Clin Neuropsychol. 1999; 13(3):348-58. [PubMed: 10726605]

19. Benedict RH, Schretlen D, Groniger L. Revision of the Brief Visual-Spatial Memory Test: studies of normal performance, reliability, and validity. Psychol Assess. 1996; 8:145-53.

20. Vega A. Use of Purdue pegboard and figger tapping performance as a apid screening test for brain damage. J Clin Psychol. 1969; 25:255-8. [PubMed: 5803245]

21. Gallagher D, Nies G, Thompson LW. Reliability of the Beck Depression Inventory with older adults. J Consult Clin Psychol. 1982; 50(1):152-3. [PubMed: 7056913]

22. Gallagher D, Breckenridge J, Steinmetz J, Thompson L. The Beck Depression Inventory and Research Diagnostic Criteria: congruence in an older population. J Consult Clin Psychol. 1983; 51(6):945-6. [PubMed: 6655109] 
23. Kendall PC, Finch AJ Jr, Auerbach SM, Hooke JF, Mikulka PJ. The State-Trait Anxiety Inventory: a systematic evaluation. J Consult Clin Psychol. 1976; 44(3):406-12. [PubMed: 932270]

24. Frerichs RJ, Tuokko HA. Reliable change scores and their relation to perceived change in memory: implications for the diagnosis of mild cognitive impairment. Arch Clin Neuropsychol. 2006; 21(1): 109-15. [PubMed: 16216465]

25. Jacobson NS, Truax P. Clinical significance: a statistical approach to defining meaningful change in psychotherapy research. J Consult Clin Psychol. 1991; 59(1):12-9. [PubMed: 2002127]

26. Jenkins VA, Bloomfield DJ, Shilling VM, Edginton TL. Does neoadjuvant hormone therapy for early prostate cancer affect cognition? Results from a pilot study. BJU Int. 2005; 96(1):48-53. [PubMed: 15963119]

27. Chertkow H, Massoud F, Nasreddine Z, et al. Diagnosis and treatment of dementia: 3. Mild cognitive impairment and cognitive impairment without dementia. CMAJ. 2008; 178(10):127385. [PubMed: 18458258]

28. Tierney MC, Yao C, Kiss A, McDowell I. Neuropsychological tests accurately predict incident Alzheimer disease after 5 and 10 years. Neurology. 2005; 64(11):1853-9. [PubMed: 15955933]

29. Spaan PE, Raaijmakers JG, Jonker C. Early assessment of dementia: the contribution of different memory components. Neuropsychology. 2005; 19(5):629-40. [PubMed: 16187881]

30. Ivnik RJ, Smith GE, Petersen RC, et al. Diagnostic accuracy of four approaches to interpreting neuropsychological test data. Neuropsychology. 2000; 14(2):163-77. [PubMed: 10791857]

31. Kayl AE, Wefel JS, Meyers CA. Chemotherapy and cognition: effects, potential mechanisms, and management. Am J Ther. 2006; 13(4):362-9. [PubMed: 16858172]

32. Zehnder AE, Blasi S, Berres M, Spiegel R, Monsch AU. Lack of practice effects on neuropsychological tests as early cognitive markers of Alzheimer disease? Am J Alzheimers Dis Other Demen. 2007; 22(5):416-26. [PubMed: 17959877]

33. Salminen E, Portin R, Korpela J, et al. Androgen deprivation and cognition in prostate cancer. $\mathrm{Br} \mathbf{J}$ Cancer. 2003; 89(6):971-6. [PubMed: 12966411]

34. Green HJ, Pakenham KI, Headley BC, et al. Quality of life compared during pharmacological treatments and clinical monitoring for non-localized prostate cancer: a randomized controlled trial. BJU Int. 2004; 93(7):975-9. [PubMed: 15142146]

35. Mohile SG, Bylow K, Dale W, et al. A pilot study of the vulnerable elders survey-13 compared with the comprehensive geriatric assessment for identifying disability in older patients with prostate cancer who receive androgen ablation. Cancer. 2007; 109(4):802-10. [PubMed: 17219443] 
Table 1

Baseline patient and disease characteristics.

\begin{tabular}{ll}
\hline Age (median and range) & $71(51-87)$ \\
Education (median and range) & $15(8-20)$ \\
Gleason (median and range) & $7(4-8)$ \\
Ethnicity & $66 \%$ Caucasian; 34\% African American \\
Marital status & $85 \%$ married \\
Disease status & $85 \%$ biochemical relapse; $15 \%$ overt metastases \\
\hline
\end{tabular}




\section{Table 2}

Mean raw score (SD) at Time 1 and percent with baseline impairment.

\begin{tabular}{|c|c|c|c|}
\hline Domain & Neurocognitive test & Time $1(n=32)$ & $\%$ IMP \\
\hline \multirow[t]{3}{*}{ Verbal memory } & Total learning & $19.10(4.79)$ & 42 \\
\hline & Delayed recall & $5.74(2.82)^{a}$ & 42 \\
\hline & Recognition hits & $10.48(1.75)$ & 36 \\
\hline \multirow[t]{3}{*}{ Visual memory } & Total learning & $16.74(7.95)$ & 33 \\
\hline & Delayed recall & $6.74(3.73)$ & 29 \\
\hline & Recognition hits & $5.60(.85)$ & 33 \\
\hline Visuospatial & RCF copy & $28.02(6.23)$ & $19^{b}$ \\
\hline \multirow[t]{3}{*}{ Executive } & TMT-A & $54.10(32.48)$ & 24 \\
\hline & TMT-Test-B & $121.42(57.09)$ & 19 \\
\hline & Digit span & $10.41(3.09)$ & 03 \\
\hline \multirow[t]{2}{*}{ Language } & Phonemic fluency & $34.87(10.6)$ & 16 \\
\hline & Semantic fluency & $16.13(4.48)$ & 10 \\
\hline \multirow[t]{4}{*}{ Motor } & Pegs dominant & $109.10(33.83)$ & 90 \\
\hline & Pegs nondominant & $120.45(46.17)$ & 66 \\
\hline & Tapping dominant & 39.5310 .07 & 14 \\
\hline & Tapping nondominant & 39.0314 .82 & 04 \\
\hline \multirow[t]{3}{*}{ Emotional } & BDI & $4.73(5.53)$ & 03 \\
\hline & State anxiety & $34.17(10.85)$ & 32 \\
\hline & Trait anxiety & $31.93(9.37)$ & 23 \\
\hline
\end{tabular}

RCF is Rey Complex Figure Test, TMT is Trail-Making Test, and BDI is Beck Depression Inventory.

${ }^{a}$ Mean >1.5 standard deviations below age- and education-adjusted population reference mean (references in Section 2.2).

${ }^{b}$ One missing data point. 
Table 3

Cognitive performances over time.

\begin{tabular}{llrrr}
\hline Domain & Neurocognitive test & Time 1 $(\boldsymbol{n}=\mathbf{2 1})$ & Time 2 $(\boldsymbol{n}=\mathbf{2 1})$ & $\boldsymbol{p}$-Value \\
\hline Verbal memory & Total learning & $19.10(4.79)$ & $19.14(6.78)$ & 1.00 \\
& Delayed recall & $5.74(2.82)^{*}$ & $5.43(3.44)$ & .80 \\
& Recognition hits & $10.48(1.75)$ & $10.67(1.62)$ & .50 \\
Visual memory & Total learning & $16.74(7.95)$ & $17.71(9.17)$ & .19 \\
& Delayed recall & $6.74(3.73)$ & $6.74(3.62)$ & .46 \\
& Recognition hits & $5.60(.85)$ & $5.71(.46)$ & .25 \\
Visuospatial & RCF copy & $28.02(6.23)$ & $29.2(6.91)$ & .78 \\
Executive & TMT-A & $54.10(32.48)$ & $43.95(27.03)$ & .16 \\
& TMT-Test B & $121.42(57.09)$ & $109.37(50.49)$ & .10 \\
Language & Digit span & $10.41(3.09)$ & $10.47(2.57)$ & .92 \\
& Phonemic fluency & $34.87(10.6)$ & $39.62(11.88)$ & .06 \\
Motor & Semantic fluency & $16.13(4.48)$ & $16.90(3.74)$ & .44 \\
& Pegs dominant & $109.10(33.83)$ & $97.60(22.57)$ & .13 \\
& Pegs nondominant & $120.45(46.17)$ & $99.30(29.16)$ & .003 \\
& Tapping dominant & 39.5310 .07 & $42.59(9.87)$ & .28 \\
& Tapping nondominant & 39.0314 .82 & $40.02(8.54)$ & .33 \\
& BDI-2 & $4.73(5.53)$ & $7.00(7.94)$ & .02 \\
& State anxiety & $34.17(10.85)$ & $35.14(11.46)$ & .93 \\
& Trait anxiety & $31.93(9.37)$ & $33.76(10.90)$ & .75 \\
\hline
\end{tabular}

RCF is Rey Complex Figure Test, TMT is Trail-Making Test, and BDI-2 is Beck Depression Inventory. 


\section{Table 4}

Specific domain based RCI*.

\begin{tabular}{llr}
\hline Cognitive domain & Decline & Improvement \\
\hline Verbal memory & $4(19 \%)$ & $5(24 \%)$ \\
Visual memory & $2(10 \%)$ & $2(10 \%)$ \\
Visuospatial & $5(24 \%)$ & $10(48 \%)$ \\
Executive functioning & $8(38 \%)$ & $6(29 \%)$ \\
Language & $3(14 \%)$ & $2(10 \%)$ \\
\hline
\end{tabular}

Verbal memory = HVLT-delayed recall; visual memory = HVLT-delayed recall; visuospatial $=$ RCF copy; executive $=$ TMT-B language $=$ fluency

Reference for RCI calculations is Jacobson and Truax [25]. 
Table 5

Cognitive performance of those with intact versus impaired cognitive functioning at baseline (Time 1) and 6 months (Time 2).

\begin{tabular}{|c|c|c|c|c|}
\hline Domain and test & Intact Time 1 & Intact Time 2 & Impaired Time 1 & Impaired Time 2 \\
\hline \multicolumn{5}{|l|}{ Verbal memory } \\
\hline Total learning & 20.60 & 18.70 & 17.82 & 19.55 \\
\hline Delayed free recall & 7.44 & 06.10 & 4.00 & 4.81 \\
\hline Recognition hits & 11.20 & 11.10 & 9.55 & 10.27 \\
\hline \multicolumn{5}{|l|}{ Visual memory } \\
\hline Total learning & 20.80 & 21.40 & 10.81 & 14.36 \\
\hline Delayed free recall & 8.80 & 8.30 & 3.90 & 5.18 \\
\hline Recognition hits & 5.60 & 5.80 & 5.27 & 5.63 \\
\hline \multicolumn{5}{|l|}{ Visuospatial } \\
\hline Rey Complex Figure Test & 30.33 & $32.94^{*}$ & 26.45 & 24.85 \\
\hline \multicolumn{5}{|l|}{ Executive } \\
\hline Trail Making Test-A & 38.62 & 35.38 & 57.18 & 48.56 \\
\hline Trail Making Test-B & 113.70 & 103.80 & 127.71 & 92.43 \\
\hline Digit span & 10.67 & 10.66 & 10.40 & 10.30 \\
\hline \multicolumn{5}{|l|}{ Language } \\
\hline Phonemic fluency & 39.22 & $46.56^{* *}$ & 33.73 & 34.73 \\
\hline Semantic fluency & 17.67 & 18.22 & 14.73 & 15.46 \\
\hline \multicolumn{5}{|l|}{ Motor } \\
\hline Groove pegs dominant & 97.80 & 99.50 & 118.80 & 95.70 \\
\hline Groove pegs nondominant & 105.11 & 90.56 & 143.70 & 110.40 \\
\hline Tapping dominant & 42.66 & 42.07 & 36.81 & 42.46 \\
\hline Tapping nondominant & 38.90 & 40.35 & 34.78 & 38.17 \\
\hline \multicolumn{5}{|l|}{ Emotional } \\
\hline Beck Depression Inventory & 1.80 & 4.10 & 7.90 & 9.90 \\
\hline State Trait (State) & 34.67 & 31.56 & 36.80 & 39.10 \\
\hline State Trait (Trait) & 31.56 & 31.11 & 37.00 & 38.22 \\
\hline
\end{tabular}

